International Journal of Engineering \& Technology, $7(2.8)(2018)$ 139-144
International Journal of Engineering \& Technology
SPC
Website: www.sciencepubco.com/index.php/IJET
Research Paper

\title{
Imaging of bone using infrared radiation
}

\author{
R. Swetha Arulmozhi ${ }^{1 *}$, Mahima Vaidya ${ }^{1}$, S. Sahana ${ }^{1}$, S. Srinithi ${ }^{1}$, T. Rajalakshmi ${ }^{1}$ \\ ${ }^{1}$ Department of Biomedical Engineering, SRM University, Kattankulathur, India, 603203 \\ *Corresponding author E-mail: srinithisridhar226@gmail.com
}

\begin{abstract}
Imaging of bone helps to identify cortical abnormalities and associated deformities. This paper aims at providing a simple device that uses non ionizing radiations from a near infrared source which can detect changes in bone shape or any related fractures. The circuit is very simple and of relatively low cost. As per the data health in India, it is seen that $30 \%$ of the total population is prone to low bone density. This is an alarming situation that led to design of this paper. The device comprises of 17 NIR LED (850 nm) and c170 Logitech 5MP web camera, with its IR filter removed. The LED array is used as the source and the camera as the detector. The light beam from the source is focused on the desired region of the skin. The source and the camera are positioned mutually perpendicular to the region of the skin to be imaged for acquisition of a perfect image of bone. The image obtained is further processed by contrast enhancement and adaptive histogram equalization. The enhanced image is studied further for bone fractures, abnormalities, etc. An attempt is also made to focus on extending this technique as a screening tool for persons affected with Fibromyalgia.
\end{abstract}

Keywords: Bone Imaging; Near Infrared; Contrast Enhancement; Fibromyalgia; IR Filter.

\section{Introduction}

Bone is a part of the vertebrate skeleton. The hard layer of bones is called compact bone. The outer layer provides a smooth and dense presentation, and covers $80 \%$ of bone entirety of an adult. Narrow formations of osteoblasts create a sporadic arrangement of spaces, known as trabeculae. [1]Altered osteoblasts become lining cells, creating a shield on the surface of the bone. The hardened matrix of tissue has a biological constituent called ossein and an inorganic part of bone mineral composed of various salts. Bone matrix is made up of $90 \%$ and $95 \%$ collagen fibres, and the rest is ground material. Collagen filaments provide bone with its ductile stability, and the distributed crystals of hydroxyapatite give bone its compressive strength. [1]

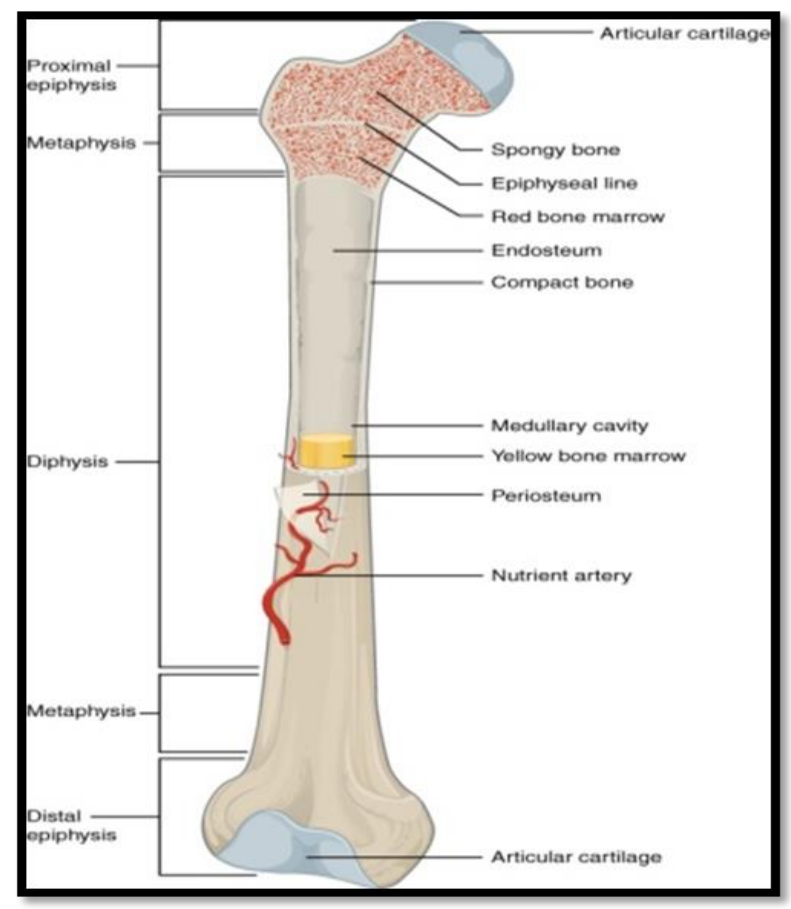

Fig. 1: Structure of Bone [1].

Fig 1 shows the structure of bone, along with its various parts. Infrared radiation is part of electromagnetic spectrum, having wavelengths greater than that of visible range and hence, is not visible. It begins from the edge of the visible spectrum at $700 \mathrm{~nm}$ to $1000000 \mathrm{~nm}$. [2] An infrared camera is a non-contact appliance that identifies infrared heat and transforms it into an electronic signal, which is then prepared to produce a thermal equivalent on a video monitor and perform temperature calculations. An IR camera embodies an optical method that targets IR energy on a 
specialized detector that consists of many detector pixels aligned in a grid. Every pixel of the sensor array addresses the IR concentrated on it, resulting in an electronic signal. [3]

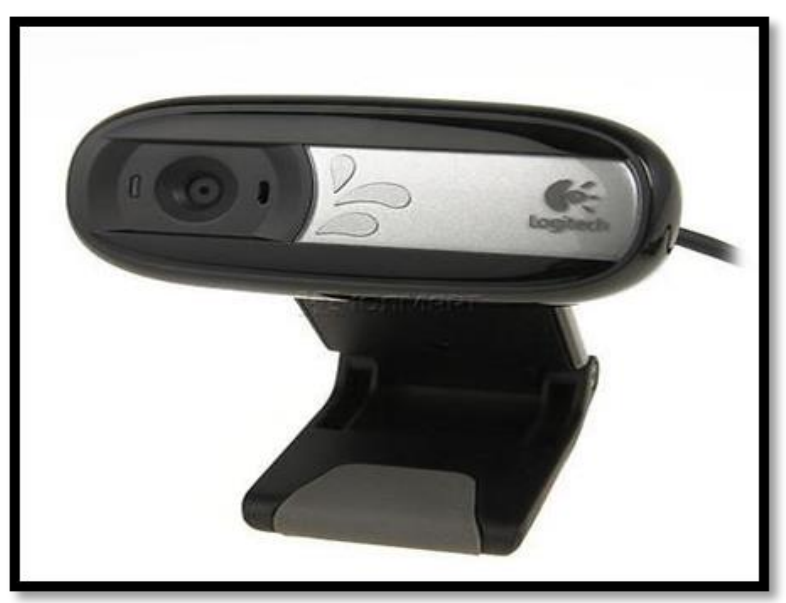

Fig. 2: C170 Logitech 5MP Webcam (https://www.ebay.com/p/LogitechC170-Web-Cam/153235870)

Fig 2 shows the webcam used as the detector to capture the image. Non-ionizing radiation is a class of electromagnetic radiation which cannot conduct substantial photon energy for ionisation of atoms or molecules. The radiation consists of ample energy for stimulation, which requires elaborate radiological preservation measures. The advantage of IR imaging over $\mathrm{X}$ rays is that, they are non-ionizing, thereby causing less harm to the tissues. [4]

Yi-Chong Zeng et al in their study proposed the usage of AHA in enhancing image contrast. The suggested procedure is carried out according to the principles of weighted histogram separation and gray-level grouping. It improves the contrast of the local detail and reduces the risks of group density and blocky effect. Also, the chief advantage of AHA technique is that, it can counteract the over-enhancement of contrast. The laboratory result shows that adaptive histogram adjustment has commendable contrast affectability, in comparison to the five current approaches. Aspiring to advance the image contrast, the above technique is considered on the basis of histogram separation structure, commonly known as WHS. In this analysis of AHA, there are two main concerns i.e, blocky effect and group density. When weighted histogram separation is applied on low-contrast copy, the blocky effect will appear. To crack this dilemma, two methods are used, namely histogram redistributing and histogram merging. There are four basic procedures for this Adaptive histogram technique that includes: histogram separation, histogram adjustment, local contrast improvement and image synthesis. Thus, AHA helps in improving the image contrast and at the same time prevents the blocky effects from the image. The completion of the experiment shows that the approach is applicable for gray-level image and colour image enhancement.

Wang Lingyu et al investigated two IR imaging techniques, farinfrared thermography and near-infrared imaging, to acquire images for biometric purposes. The imaging dogmas for both are studied in detail. X-ray and ultrasonic scanning is adopted in all medical practices for obtaining vascular images. The key challenge in biometric system is to obtain these images in a quick and non-invasive fashion. This paper centralizes on mainly two IR technologies: Far-Infrared (FIR) and Near-Infrared (NIR). IR imaging in these two areas gives a junction-less, non-invasive data procuration method and needs no inoculation of any factors into the blood. Special attributes of IR radiation is that, the incident IR light can diffuse into the tissue to $3 \mathrm{~mm}$ depth approximately. Therefore, by directing the IR beam at the required region of interest, an image can be obtained using a CCD camera having an attached IR filter. There exists a "medical spectral window" which extends from 700 to $900 \mathrm{~nm}$. Therefore, the wavelength of the IR light ejecting from the source is chosen to be in the near infrared region with wavelength around $850 \mathrm{~nm}$. This avoids rejected hin- drance from the infrared radiation given out by the body and its surrounding.

Aykut Eken et al used near infrared spectroscopy signals (NIRS) to detect fibromyalgia. Exact reason of this syndrome has not been known yet. In this study, painful stimuli was applied with transcutaneous electrical nerve stimulation (TENS) to participants' right and left hand. At the same time, functional near infrared spectroscopy signals (fNIRS) was acquired and support vector machine (SVM) method was applied by using polynomial functions in different orders to the feature vector created. According to the results, by using fNIRS signals in post central gyrus and angular gyrus and in inferior parietal gyrus, discrimination between FM patients and healthy controls could be achieved by approximately $70 \%$ performance.

S. K. Mitra et al introduced the usage of non-linear filters for image enhancement. This is on the basis of Teager's algorithm. A conjectural reasoning has revealed that a type of nonlinear filter functions as a local-mean-weighted high pass filter. Another type of nonlinear filter has been refined which also works as a localmean-weighted bandpass filter. The filtered image thus obtained from one of those nonlinear filters is combined with the original image, to acquire image enhancement. By using this technique, a high standard of enhancement has been attained for the images. The aim of the proposed study is to image bone tissue with better clarity using infrared radiation.

\section{Electrical equivalence circuit for infrared source-detector system}

The circuit diagram consists of six 850nm IR LEDs arranged in a parallel fashion. The arrangement is connected to two $330 \Omega$ resistors. A 9V battery is used as the power source. The circuit was simulated using Multisim software in order to confirm the design configuration.

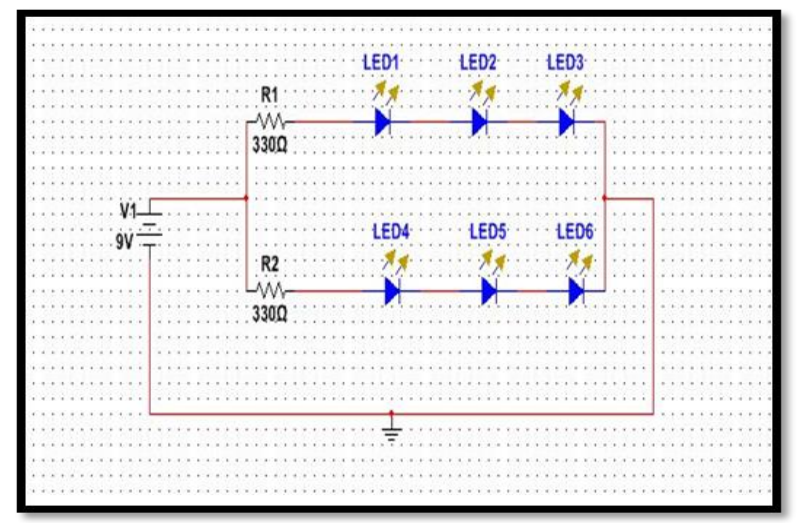

Fig. 3: Electrical Equivalence Circuit of NIR Source.

Fig 3 shows the electrical equivalence circuit of NIR source. The IR filter is removed from the webcam prior to the image capture. A variety of resistors and IR LEDs are used. The LEDs are arranged in an array in order to focus the source on the body part to be imaged. A battery of suitable voltage is used to power the device. A webcam is used to obtain the image and it is viewed using the camera software. A toggle switch, along with a light indicator, is used to operate the source. 17 infrared LEDs of $850 \mathrm{~nm}$ type are arranged in an array to form the light source. The number of LEDs selected depends on the level of penetration required. A C170 Logitech 5MP web camera is chosen to obtain the image.

$1^{\text {st }}$ Model: The first model was built on a breadboard and had 6 IR LEDs of $850 \mathrm{~nm}$ type and 2 resistors of $330 \Omega$. A battery of $9 \mathrm{~V}$ was used. The source and the detector were moved in various directions to obtain a partial image of the body part (knee, elbow, foot). 


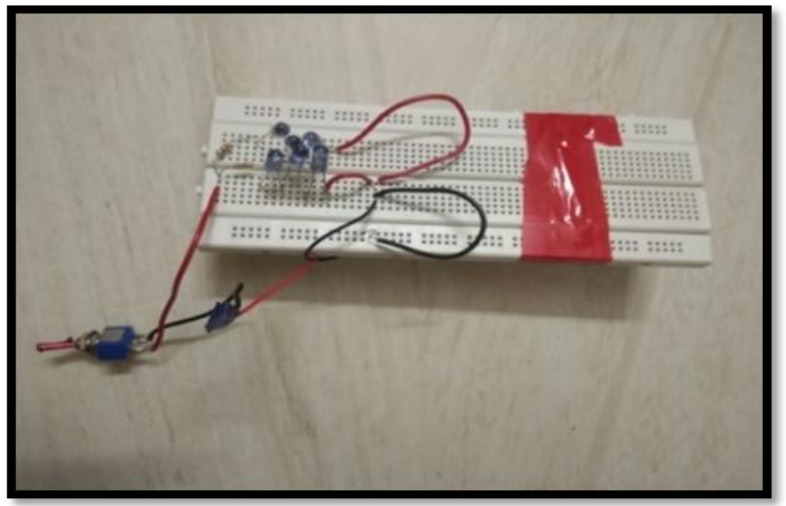

Fig. 4: Breadboard Model.

Fig 4 is the basic breadboard model with 6 LEDs and 2 resistors. $2^{\text {nd }}$ Model: The second model was built on a PCB board and had 12 LEDs of $850 \mathrm{~nm}$ type and 8 resistors of $330 \Omega$. A battery of $4 \mathrm{~V}$ was used. The detector was kept stationary, while the source was moved in different directions.

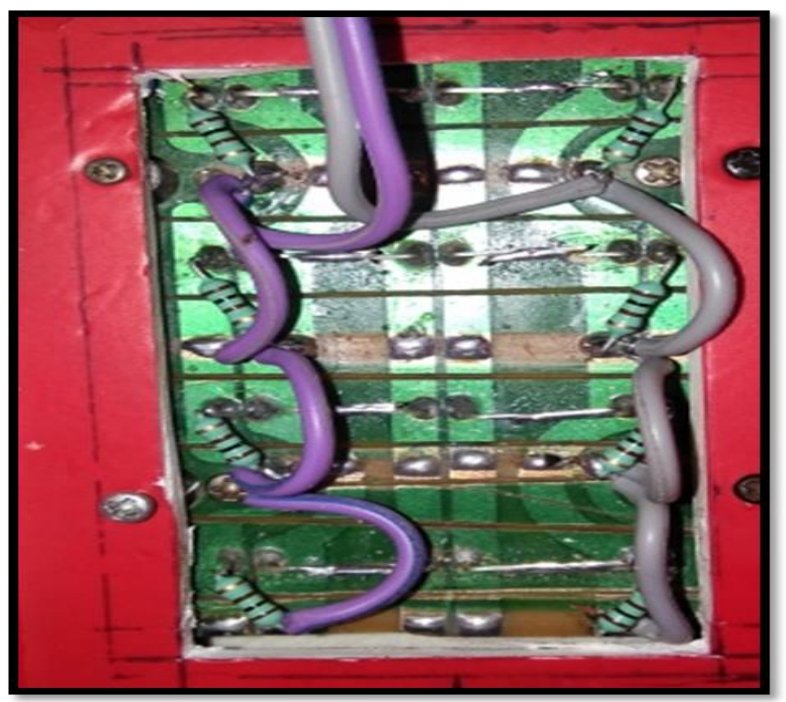

Fig. 5: Resistor-LED Setup.

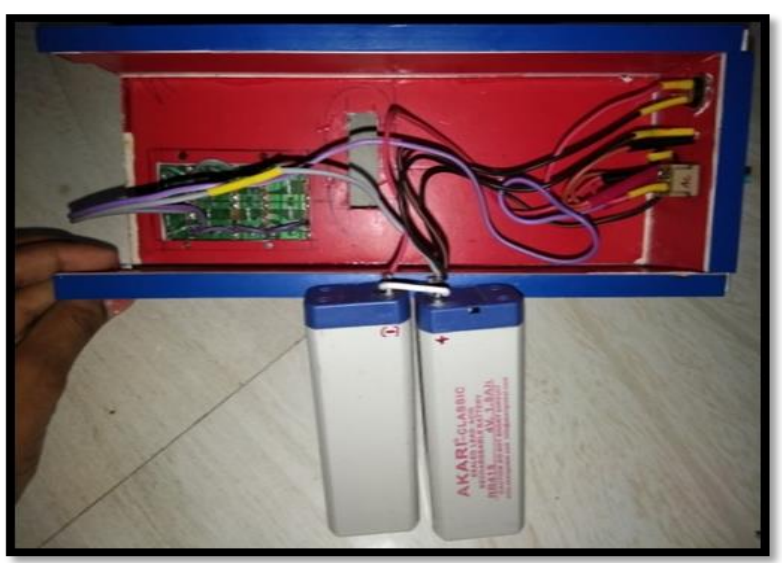

Fig. 6: Overall Circuit.

Fig 5 shows the resistor-LED setup on a PCB and Fig 6 depicts the overall circuit of the $2^{\text {nd }}$ model.

$3^{\text {rd }}$ Model: The final model is built on a PCB board and has 17 LEDs of $850 \mathrm{~nm}$ type and 8 resistors of $330 \Omega$. A battery of $4 \mathrm{~V}$ was used. The source was kept stationary, while the detector was moved in various directions. It is important to note that the source and detector are placed mutually perpendicular to each other in order to obtain a clear image.

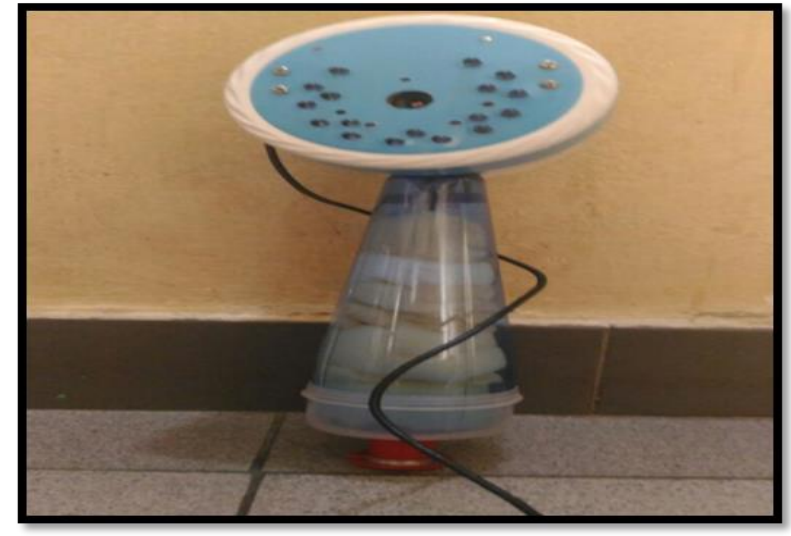

Fig. 7: Final Model Having 17-LED Circular Array.

Fig 7 shows the final model of the NIR source with 17 LEDs.

\section{Proposed methodology}

It is well known that near infrared radiation has low attenuation losses. It has a wavelength of $0.75-1.4 \mu \mathrm{m}$ and a frequency of $214-$ $400 \mathrm{THz}$ and image intensifiers prove to be sensitive to that region of energy spectrum. Contrast enhancement is an often used technique in the field of image processing. It is a useful technique for processing various scientific images such as X-Ray images and satellite images. Contrast enhancement is also useful in improving and capturing the finer details in photographs that are either over or under-exposed. [2 5 6]

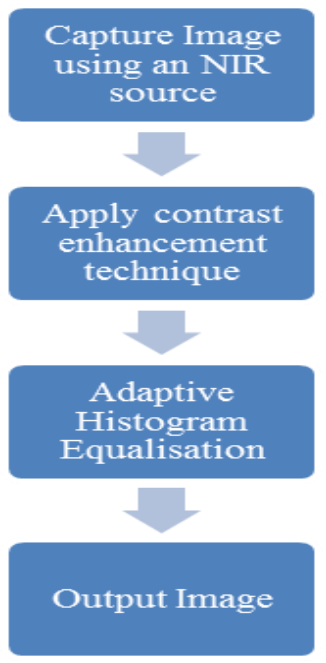

Fig. 8: Block Diagram of Proposed Work.

Fig 8 is a flowchart of the proposed work.

The IR filter in the webcam, used to capture the image, is removed in order to allow IR radiation to pass through it. The Infrared source contains 17 LEDs of $850 \mathrm{~nm}, 8$ resistors of $330 \Omega$, and a $4 \mathrm{~V}$ battery. The source (IR source) and the detector (camera) are held perpendicular ( 90 degrees) to each other in order to focus the source at the area to be imaged. The IR rays penetrate the surface of the body for a period of about 6 minutes and the image of bone tissue is obtained after 3 minutes. The body part can be imaged from top and side views. On consulting the orthopaedic doctor, images obtained were confirmed as bone tissue.

The contrast enhancement technique that is applied to the obtained images is called Adaptive Histogram Equalisation method. This method, also known as the AHE, a computer image processing technique, is used in enhancing contrast of images. It proves to be a good method for boosting the local contrast and the definitions of edges in every region of the image. [7]

The images obtained initially are hazy and unclear. While using 6 LEDs, there isn't sufficient brightness to capture the image. Also, 
the source and detector are moved continuously. While using 12 LEDs, the source is in motion while the detector is stationary. Only by using 17 LEDs, required brightness is obtained while moving the camera and keeping the IR array source stationary. It is necessary to note that the source and detector are mutually perpendicular to each other while capturing the images. The images are obtained for the knee, elbow and, foot. After repeated experimentation by changing the number of LEDs and the position of the source and detector, viewing of the radius and ulna, bones of the thigh and, phalanges is possible.

\section{Results \& discussion}

Images were captured at the knee, elbow and foot regions. The following images were captured using 6 LED, 12 LED and 17 LED configurations.

Knee: The 6 LED arrangement resulted in a partial image of the knee. The source was moved in various directions along with the detector to capture the image. The time taken for image formation was approximately 45 minutes.

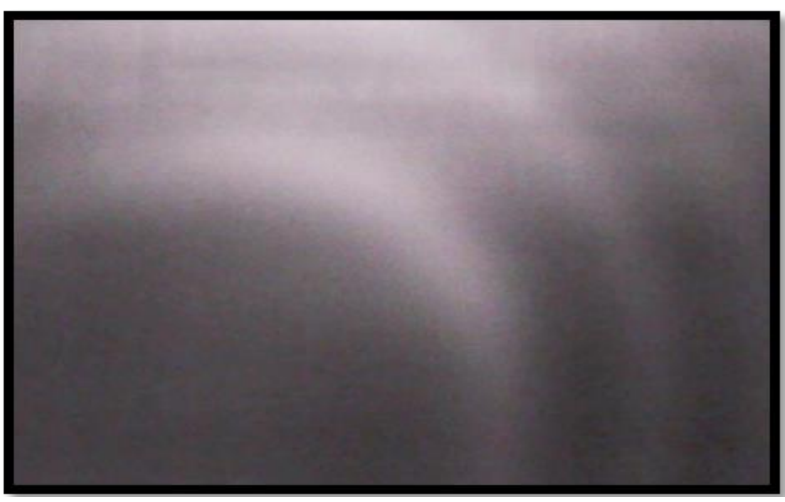

Fig. 9: 6-Led Arrangement (Knee)

Fig 9 shows the image of knee captured with the 6 LED breadboard model.

- The 12 LED configuration yielded a clearer image of the knee. The image was captured keeping the detector stationary, while the source was placed at various angles. The time taken for image formation was 15-20 minutes.

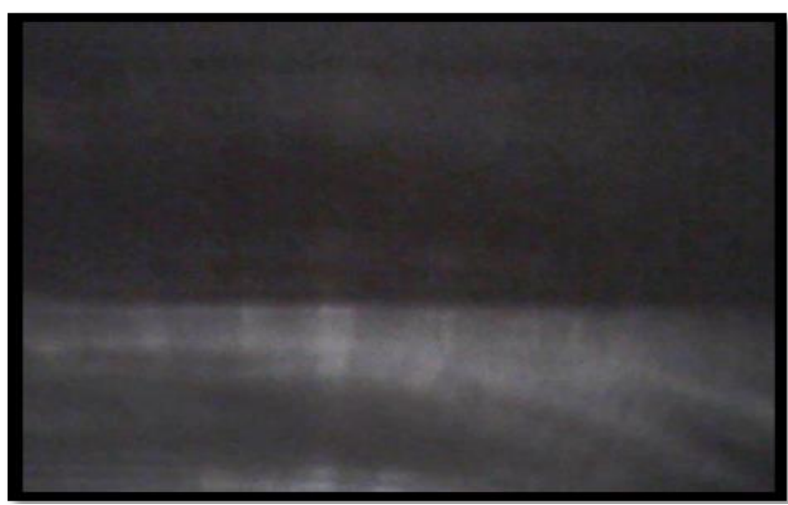

Fig. 10: 12 LED Arrangement (Knee)

Fig 10 is the image of knee captured with the 12 LED PCB model.

- The number of LEDs were increased to 17 in order to increase the brightness of the image. The IR source was kept in a stationary position and the camera was moved in different directions, while being kept at 90 degrees from the source. The time taken to obtain the image was 10 minutes.

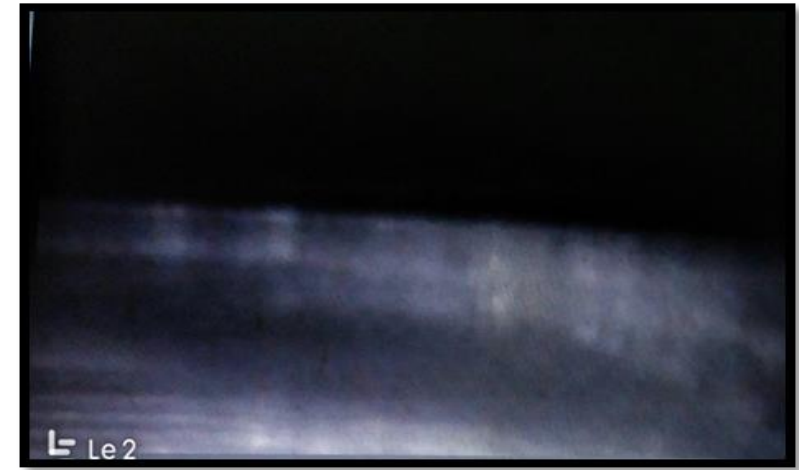

Fig. 11: 17-Led Arrangement (Knee).

Fig 11 is the image of knee captured with the 17 LED circular array.

Elbow: The 6 LED configuration resulted in the formation of a poor image with less brightness. Indistinct shapes were detected by moving the source and detector in various directions.

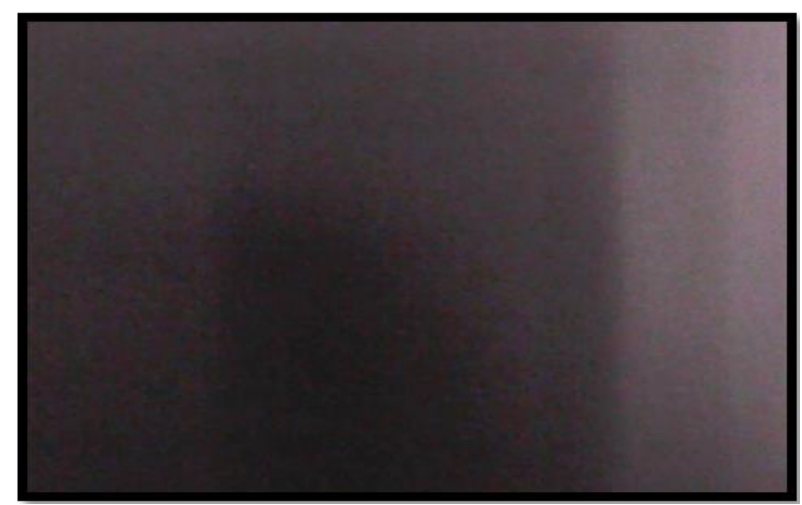

Fig. 12: 6-Led Arrangement (Elbow).

Fig 12 shows the image of elbow captured with the 6 LED breadboard model.

A 12 LED arrangement was used to obtain the image while rotating the source and keeping the detector stable.

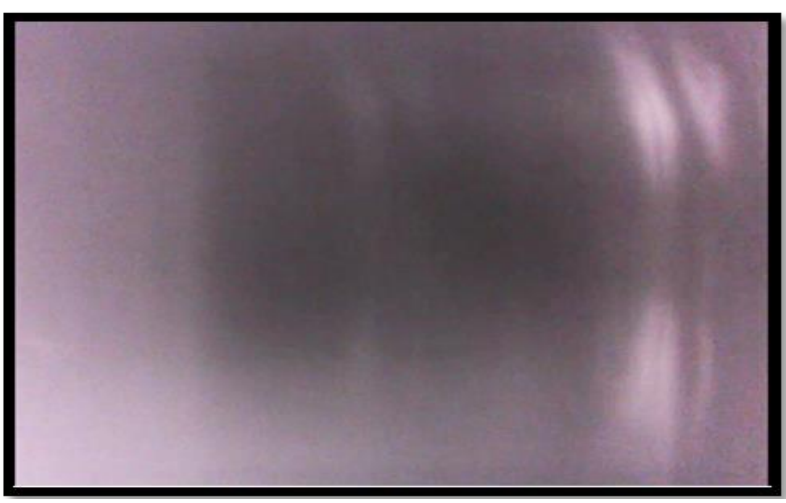

Fig. 13: 12-Led Arrangement (Elbow).

Fig 13 is the image of elbow captured with the 12 LED PCB model.

The best image was obtained while using 17 LEDs. The detector was rotated while the source was kept in stationary position 


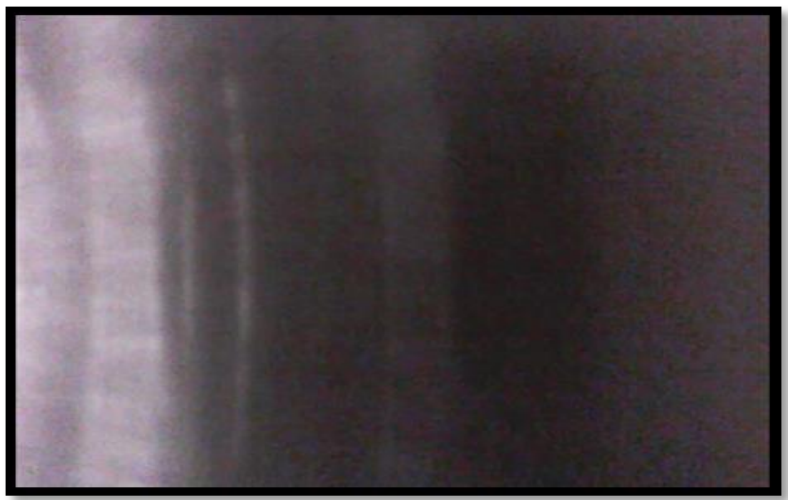

Fig. 14: 17-Led Arrangement (Elbow).

Fig 14 is the image of elbow captured with the 17 LED circular array.

Foot: 6 LEDs are arranged on a breadboard along with 2 resistors to image the foot. The top view of the foot is imaged by moving the source in different positions.

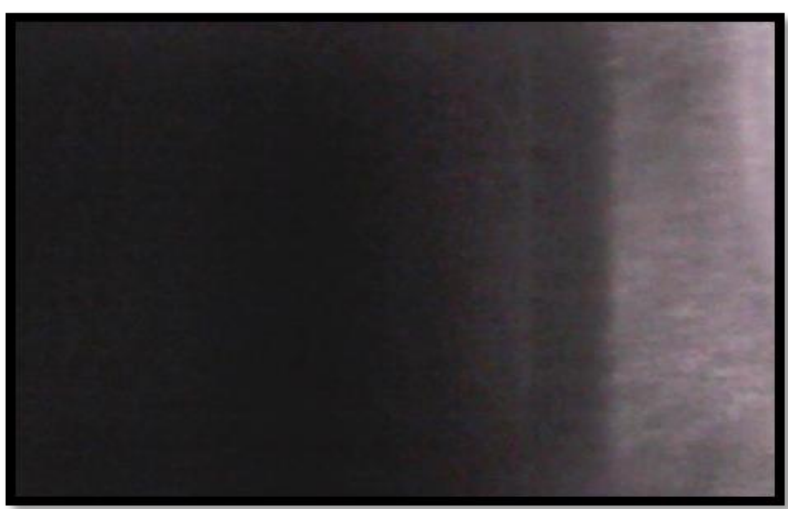

Fig. 15: 6-Led Arrangement (Foot).

Fig 15 shows the image of foot captured with the 6 LED breadboard model.

12 LEDs are arranged in a rectangular array along with 8 resistors. A faint image of the phalanges is obtained.

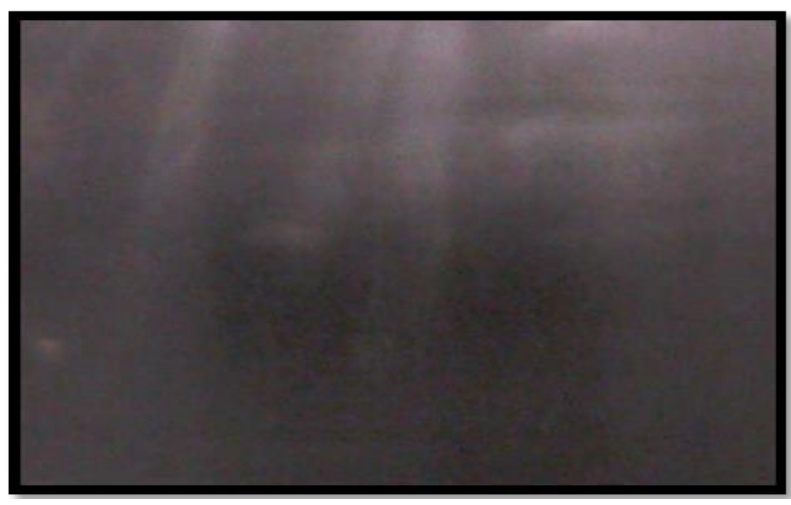

Fig. 16: 12 Led Arrangement (Foot).

Fig 16 is the image of foot captured with the 12 LED PCB model.

- 17 LEDs are arranged in a circular array to focus the amount of infrared entering the body part. A clearer image of the phalanges is obtained by moving the detector in a direction perpendicular to the source.

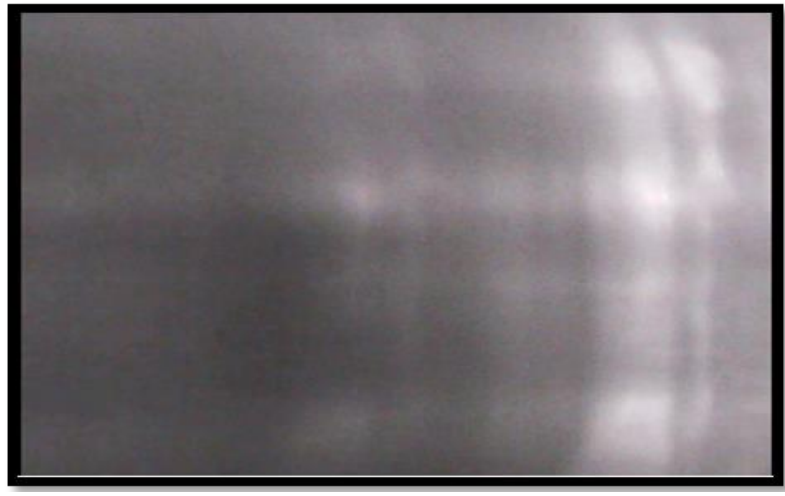

Fig. 17: 17 Led Arrangement (Foot).

Fig 17 is the image of foot captured with the 17 LED circular array.

\section{Conclusion}

One of the major limiting factors of near infrared light source is the time taken to penetrate the skin. Since the proposed device is incorporated with many LED's in a circular setup, the time taken is relatively less and the imaging is also done quickly. It is also essential to point out that the above mentioned duration was observed for a subject who was thin. Hence, it can also be concluded that the time it takes for imaging a person of normal weight or more is greater. The source and the camera has to be held in the same position for a long time which is inconvenient for both, the patient as well as the operator. Also, the whole procedure has to be done in a dark room which is a disadvantage. The transmitters and receivers (source and body part respectively) must be closely aligned. The performance will drop if the distance to the receiver is very large. The path between the source and the detector must be free of any obstacle to get an accurate image. The camera also plays an important role here. When the camera is working, any kind of disturbance (moving objects, changing camera position constant, etc.) is generally not preferred.

With further modifications and advancements in the design of the circuit, there is a possibility to image the muscle tissue along with the bone. The image can give us information about impending risk of bone fractures leading to osteoporosis. Also via this imaging, detection of Fibromyalgia can be done, which is a major challenge today to diagnose. Fibromyalgia occurs due to accumulation of ground substance in the muscle at 18 tender points in the body. Using this imaging technique, the accumulation can be detected and further treatment can be given by doctors. The most important feature of imaging is to help detect and diagnose the abnormalities as well as help in biological researches. This principle is well utilized in designing this device.

\section{References}

[1] https://en.wikipedia.org/wiki/Bone

[2] https://en.wikipedia.org/wiki/Infrared

[3] www.flir.com/corporate/display/?id=41523

[4] https://en.wikipedia.org/wiki/Non-ionizing_radiation

[5] https://broom02.revolvy.com/main/index.php?s=Near\%20infrared \&item...topic

[6] https://sites.google.com/site/5kk70gpu/assignment-s/colorconversion

[7] https://en.wikipedia.org/wiki/Adaptive_histogram_equalization

[8] W. K. Pratt. 2001. Digital image processing. John Wiely \& Sons, Inc. 3 .

[9] R. A. Hummel. 1977. Image enhancement by histogram transformation. Computer Graphics and Image Processing. 6(2). pp.184195. https://doi.org/10.1016/S0146-664X(77)80011-7.

[10] W. Frei. 1977. Image enhancement by histogram hyperbolization. CVGIP. 6. pp.286-294.

[11] Wang Lingyu and Graham Leedham. 2006. Near- and Far-Infrared Imaging for Vein Pattern Biometrics. Proceedings of the IEEE In- 
ternational Conference on Video and Signal Based Surveillance. AVSS

[12] Yi-Chong Zeng. 2009. Automatic Local Contrast Enhancement using Adaptive Histogram adjustment. Proceedings of the IEEE Multimedia and Expo.

[13] S. K. Mittra et al. 1991. A new class of nonlinear filters for image enhancement. Acoustics, Speech and Signal Processing. https://doi.org/10.1109/ICASSP.1991.150915.

[14] Sunil Deshpande et al. 2011. A Control Engineering Approach for Designing an Optimized Treatment Plan for Fibromyalgia. American Control Conference.

[15] Aykut Eken et al. 2015. Classification of fibromyalgia syndrome using NIR signals. Medical technologies national conference.

[16] Xiaobin Daia et al. A Fast Vein Display Device Based on the Camera-projector System. Sch. Of Optoelectronics. Sch. of Life Science. Beijing Institute of Technology.

[17] Yi-chong Zeng. Automatic local contrast enhancement using adaptive histogram adjustment. Institute of information science.

[18] Carolina Pieroni Andrade*, Antonio Roberto Zamunér, Meire Forti. Aparecida Maria Catai, Patrícia, Driusso, Ester da Silva. 2014. Association between Baroreflex Function and Pressure Pain. Threshold in Women with Fibromyalgia. 8th Conference of the European study group on cardiovascular oscillations.

[19] A Control Engineering Approach for Designing an Optimized Treatment Plan for Fibromyalgia Sunil Deshpande, Naresh N. Nandola, Daniel E. Rivera and Jarred Younger, 2011 American Control Conference on O'Farrell Street, San Francisco, CA, USA, June 29 July 01, 2011.

[20] Evren Arslan, Sedat Yıldız, Etem Köklükaya, Yalçın Albayrak. Classification of Fibromyalgia Syndrome by Using Fuzzy Logic Method.

[21] Tamara L.Troy, Suresh N.Thennadil. 2001. Optical properties of human skin in the near infrared wavelength range of 1000 to 2200 nm, J biomedical optics, 6 (2):167-176 https://doi.org/10.1117/1.1344191.

[22] Fatma vatansever, Michael R.Hamblin. 2012. Far infrared radiation: its biological effects and medical applications. Harvard Medical School.

[23] Aton M.Holzer, MD, Craig A.Elmets, MD. 2010. The other end of the rainbow: infrared and skin, J Invest Dermatol. 130(6): 14961499. https://doi.org/10.1038/jid.2010.79.

[24] Theodore. A. Henderson et al. 2015. Near-infrared photonic energy penetration. Neuropsychiatr Dis Treat. 11: 2191-2208.

[25] W. E. Mayo et al. 2001. Infrared Microscopic Imaging of Bone: Spatial Distribution of $\mathrm{CO}_{3}{ }^{2-}$. Journal of Bone and Mineral Research. 16(5): 893-900. https://doi.org/10.1359/jbmr.2001.16.5.893.

[26] Dr. Seetaiah Kilaru, Hari Kishore K, Sravani T, Anvesh Chowdary L, Balaji T "Review and Analysis of Promising Technologies with Respect to fifth Generation Networks", 2014 First International Conference on Networks \& Soft Computing, ISSN:978-1-47993486-7/14, pp.270273, August 2014.

[27] T. Padmapriya and V. Saminadan, "Improving Throughput for Downlink Multi user MIMO-LTE Advanced Networks using SINR approximation and Hierarchical CSI feedback", International Journal of Mobile Design Network and Innovation- Inderscience Publisher, ISSN : 1744-2850 vol. 6, no.1, pp. 14-23, May 2015.

[28] S.V.Manikanthan and K.srividhya "An Android based secure access control using ARM and cloud computing", Published in: Electronics and Communication Systems (ICECS), 2015 2nd International Conference on 26-27 Feb. 2015, Publisher: IEEE, https://doi.org/10.1109/ECS.2015.7124833. 